\title{
Phytomedicine and nutrition that help fight cancer
}

Dragan Jovanov ${ }^{1^{*}}$

${ }^{1}$ Mit University - Skopje, Macedonia, Environmental Resources and Food Security Management.

*Corresponding Author: Dragan Jovanov, Mit University - Skopje, Macedonia, Environmental Resources and Food Security Management, Tel: +38970867356 E-mail: daci.j@hotmail.com

Citation: Dragan Jovanov, (2017) Phytomedicine and nutrition that help fight cancer, Int J Nutr Sci \& Food Tech 3:1, 19-25.

Copyright: (C) Dragan Jovanov, This is an open-access article distributed under the terms of the Creative Commons Attribution License, which permits unrestricted use, distribution, and reproduction in any medium, provided the original author and source are credited.

Received February 21, 2017; Accepted March 1, 2017; Published March 17, 2017.

Phytomedicine is modern and science based herbal medicine at its very best. Phytomedicine applies scientific research and the highest professional standards to the practice of herbal medicine. It takes its name from the word used in other parts of Europe, where plant based medicines continue to be provided by doctors and pharmacists as 'phytomedicines'. Qualified Phytotherapists use herbs from around the world, and choose the most effective herbs from the best quality sources. Phytomedicine encourages and adapts to a recent worldwide growth in scientific information on plant drugs and their effective and safe use. The methods used to evaluate plant based medicines are similar to those used by orthodox medicine. Yet, a herb contains many active chemicals, unlike conventional drugs, which focus on specific chemicals. Hence botanical or herbal medicines may combine several actions to support the body's health. Botanical drugs are safe for most conditions. The increase in clinical trials in this area has helped to include these compounds in evidence-based practice. Section 12 (1) of the 1968 Medicines Act permits Phytotherapists to make and dispense herbal remedies to individual patients following a consultation. Phytomedicine, also called Botanical/Plant Medicine, differs from Chinese Herbalism. Phytotherapists use medicinal plants, which grow mainly in the western hemisphere, and do not combine their therapy with acupuncture. The word 'phyto' derives from the Greek work plant; hence it means plant based medicine. Phytomedicine is rooted in scientific research and therefore not to be confused with homeopathy.

Phytotherapy or medical herbalism, as this is called in Europe, is the most scientifically-validated medicine in the category of natural medicine.

Eating the right kinds of foods before, during, and after cancer treatment can help the patient feel better and stay stronger. A healthy diet includes eating and drinking enough of the foods and liquids that have the important nutrients (vitamins, minerals, protein, carbohydrates, fat, and water) the body needs.

Inspiration to write this article was to help on all people around the World. Primary,we face with serious diseases that overpower the world, and increase the percentage of deaths quite large. With this book I want to help (through the mother Nature), to create appropriate medicines and treatments based on natural herbs and healthy food.

\section{Phytomedicine In Cancer Treatment}

Phytomedicine refers to the application of scientific research and the highest standards of professionalism to the herbal medicine practice. The use of phytomedicine is not new though. It has been in practice for quite a long time in fact the name phytomedicine is derived from the word used in other parts of Europe, where plant based medicines are provided by doctors and pharmacists as 'phytomedicine' to their patients. Phytomedicine is more advanced in herbal medicine as it involves scientific research to ascertain the potency of the natures given herbals before they are prescribed to be used by any patient. The doctors who use phytomedicine are called phytotherapists and they treat their patients with the best herbs that fit their king of diseases. Phytotherapists choose the best herbs from the most effective herbs ensuring that very patient get the best combination of the phytomedicine.

Phytomedicine is practiced in clinical treatment of diseases in most parts of the world and is a growing field as it involves scientific research and information on the use of plant medicinal components and their safety in use. There are methods that are used to evaluate the effectiveness of plant medicines which are similar to the evaluation of orthodox medicine. Herbs unlike the orthodox medicine that focus on given chemicals contain very many bioactive components. The phytomedicine may involve use of a combination of herbs to come up with a therapy that will heal the body and strengthen its immune system to fight diseases better. 
Phytomedicine are safe for use for many diseases and cancer is no exception. After very many clinical trials of phytomedicine the use of phytomedicine in treatment of various conditions have been recognized in most countries through the world. Section of the 1968 Medicines Act permits Phytotherapists to make and dispense herbal remedies to individual patients following a consultation.

As used in cancer treatment, Phytomedicine involves selection of plant medicines to stimulate or strengthen the body's own functions and immune system, hence support the body to heal itself. The phytotherapist knows that each cancer patient is a unique individual and hence diagnoses and assesses the needs of the cancer patient uniquely. The patient will thereafter be given a prescription of combined plant products that work best in healing them.

Some of the natural plants components that have been used in phytomedicine to treat cancer include; resveratrol, saponins, silymarin, curcumin, peach compounds, green tea extracts, coffee enemas and grape seed extracts. These phytomedicine are rich in anticancer properties and are even safer for use in cancer treatment. The use of chemotherapy in cancer treatment may fail to work and if chemotherapy is done repeatedly the tumors only become more resistant to the drugs that are supposed to be killing them. The scientists have found out that the use of natural products work better in cancer treatments as they target multiple pathways thereby inhibiting growth and spread of the cancer cells in the body. Some of this phytomedicine help in killing cancer cell by inducing apoptosis in tumors.

EGCG and GSE are two popular plant extracts that have attracted much attention in recent years due to their antioxidant, antimicrobial, anticarcinogenic, and anti-inflammatory properties.

\section{Herlbs \& Spices That Help Fight Cancer}

Fighting cancer is something our body is doing all the time, not just when one is diagnosed with the disease. Cancer is triggered by toxins in the environment and in our food, inflammatory conditions, free radicals formed in the body, hormonal actions, recurrent bacterial infections and viral diseases. When any of the above agents causes damage to a cell, and the damaged cell gets to divide forming copies of itself, a cancerous growth starts.

Our immune system tries to destroy these abnormal cells through different mechanisms. Making more killer T-cells, inducing the abnormal cells to self-destruct (apoptosis), secreting tumor necrotizing factor (TNF), and preventing the formation of new blood vessels (angiogenesis) that feed the tumor are some of them. Many bioactive compounds in our food help the body in these efforts to fight off cancer. Some culinary herbs are particularly rich in cancer preventive and cancer-fighting substances. It would seem that nature has endowed them with striking aromas and strong flavors just to grab our attention so that we'd include these potent herbs in our culinary traditions. Even sick animals seek plants with certain medicinal values to heal themselves. Let's look at some culinary and medicinal herbs and spices that can help fight cancer.

International Journal of Nutritional Science and Food Technology An Open Access Journal

\section{Turmeric}

This basic ingredient of curry powder comes from the ginger-like rhizome of an herbaceous annual Curcuma longae. The curcumin in turmeric is a very strong anti-inflammatory agent, besides having wide spectrum antimicrobial and antiparasitic properties. Its anti-cancer activity includes aiding the apoptosis of damaged cells, preventing their rapid division to form tumors, and inhibiting the development of capillaries that feed tumors.

Low incidence of certain cancers is noted in communities traditionally using turmeric as a condiment. Phase I clinical trials have shown that curcumin can indeed prevent precancerous tissues from developing into cancer. It is effective against cancers of the digestive tract, breast, and lung.

The bioavailability of curcumin is increased by cooking the turmeric powder for over 10-15 minutes. It easily binds to fats, and when heated in oil, lends its bright yellow color and pungent smell without bite to dishes.

\section{Garlic}

Garlic or Allium sativum and other Allium family vegetables such as onions, shallots, and leeks are rich in organosulfur compounds alliin, allicin, and allyl sulfides which are known to be cancer protective. They have been found to protect against stomach and colon cancers. Including more of these vegetables in the diet can reduce your risk of other cancers too.

The diallyl disulfide derived from garlic killed off leukemia cells and prevented the growth of breast, lung, and skin cancer cells in lab studies. Ajoene is another potent anti-cancer substance isolated from garlic. The mechanism of action is manifold, and includes DNA repair, slowing down of cell division and apoptosis of abnormal cells among others. World Health Organization's advice to eat a clove or two of garlic is not without valid reason.

\section{Ginger}

Ginger is the underground stem/rhizome of the tropical herb Zingiber officinale. This popular spice adds a refreshing, yet pungent kick to foods. The rhizome of ginger is used both fresh and dried. Surprisingly, the flavor of fresh ginger used in cooking and the dried spice powder used to flavor gingerbread and cookies are as different as can be. The active substances in them differ as well, fresh ginger carrying gingerol and the dry ginger having zingerone. Both are anti-inflammatory and antioxidative, reducing the risk of cancer.

In laboratory studies conducted in King Abdulaziz University, Saudi Arabia, ginger extract has been shown to inhibit cell proliferation in breast cancer. This action was targeted exclusively against abnormal cells, while normal breast cells were left alone. In mice having prostate cancer, administration of raw ginger extract reduced the tumor size to half the original size.

The phytochemicals in ginger promote apoptosis of cancer cells and have a preventive action against metastasis. Since ginger tea and extract are often recommended for reducing nausea and vomiting associated with chemotherapy, it can be an excellent adjuvant to conventional treatments for cancer. 


\section{Black Pepper}

Black pepper is the dried fruit of the vine Piper nigrum. Wars have been fought and countries captured for the sake of this spice, aptly called 'black gold.' White pepper is nothing but its skinned version. The anti-inflammatory property of black pepper has long been exploited in traditional herbal medicines, but the piperine extracted from the spice is now found to have excellent antioxidant and anti-cancer effect.

Piperine has the ability to inhibit breast cancer stem cells, particularly those of the very aggressive, non-estrogen sensitive types that do not respond to current preventive drugs tamoxifen and raloxifene. It does not have the toxic side effects of these drugs and leave the normal breast tissue intact. Not only that, piperine enhances the cancer-preventive action of curcumin and increases the bioavailability of the potent polyphenolic compound epigallocatechin-3-gallate in green tea. A daily dose of one-eighth of a teaspoon may be all you need to reap the benefits.

\section{Rosemary}

The Mediterranean shrub Rosmarinus officinalis with aromatic leaves is well known for its medicinal properties. It contains rosmarinic acid and rosemary-caffeic acid which are antiinflammatory and antioxidant, capable of reducing the oxidative stress that triggers many cancers. In a study on mice having skin cancer, rosmarinic acid reduced inflammation and suppressed the development of tumors.

The carnosol in rosemary neutralizes toxins that trigger breast cancer. The terpenes from the plant inhibit the growth and proliferation of cancer cells. When used an adjuvant to Adriamycin, it helped reduce the resistance of tumor cells this chemotherapy drug.

\section{Oregano}

Oregano or wild marjoram that goes by the name Origanum vulgare is another Mediterranean herb with proven cancer protective effect. The monoterpenoid phenolic compound carvacrol, present in oregano and many other members of the mint family help prevent the spread of cancer. Another way oregano can protect us from cancer is by reducing the formation of carcinogenic heterocyclic amines that form when animal proteins are cooked at high temperatures. Soaking the meat and fish in a marinade with oregano prior to cooking may reduce your risk of cancers triggered by HCAs.

\section{Parsley}

Petroselinum crispum or garden parsley belonging to the Apiaceae family is widely used as an herb in many indigenous cuisines across the world. The volatile oils in this herb have antioxidant action, especially myristicin which inhibit the formation of tumors and activate glutathione-S-transferase enzyme to produce glutathione, the most important antioxidant generated within our body. Luteolin is an excellent antioxidant flavonoid occurring in this herb.
The apigenin found in parsley, as well as in celery belonging to the same family, is an active cancer-fighting agent. This phytochemical induces autophagy in blood cancer cells and inhibits tumorinduced angiogenesis.

\section{Dill}

Dill, the herb Anethum graveolens from the Apiaceae family, is a popular addition to pickles where its strong antimicrobial property is put to good use. Its cancer preventive effect comes from the monoterpene anethofuran occurring in the leaves and seeds of this herb. It triggers the secretion of glutathione-S-transferase which facilitates the antioxidant action of glutathione to relieve oxidative stress caused by environmental pollutants. Limonene and carvone are two other active monoterpenes found in dill. They help prevent the DNA damage that could trigger cancers.

\section{Flaxseed}

The seeds of Linum usitatissimum, usually get lauded for their omega-3 fatty acids and for their beneficial effect on heart health. When it comes to fighting cancer, it is the lignans such as Secoisolariciresinol (SDG) that get the credit. These plant estrogens mimic the estrogen secreted in our body and down regulates its action, thus helping reduce the risk of estrogen-dependent breast cancer.

It has been reported that regular consumption of flaxseeds by a group of women has resulted in 33\% reduction in tumor growth. This gives us one more reason to include these healthy seeds in our diet.

\section{Wheatgrass}

The juice of the young shoots of the wheat grain Triticum aestivum has many health benefits, including cancer-protection. It is a detoxifier and cleans out the liver and the kidneys and purifies the blood. Wheatgrass has been used to treat leukemia too.

The plant hormone abscisic acid occurring in the wheatgrass is responsible for its anti-cancer activity. In plants, this hormone down regulates physiological processes and delays cell division. Within our body, it brings about a strong anti-inflammatory effect which is beneficial to people suffering from inflammatory bowel disease. Anecdotal evidence shows that wheatgrass juice taken daily can cure breast cancer.

\section{Aloe vera}

Aloe vera is well known for its skin protective and antiinflammatory properties. Fighting inflammation is one way to reduce the risk of cancer, but this succulent herb also contains many beneficial polysaccharides such as Acemannan. They can induce the production of Tumour Necrosis Factor that destroys cancer cells.

The immune-boosting actions of include increasing the production of interferon and promoting T-cell function.

Aloe vera can be easily included in the cancer fighting arsenal because of its easy availability and excellent safety profile. 


\section{Feverfew}

As the name indicates, Feverfew or Tanacetum parthenium has been traditionally used to reduce fever and headache. But the interest in this herb has been rekindled with the discovery that parthenolide derived from it can destroy leukaemia cells.

As an excellent anti-inflammatory agent, parthenolide can reduce the risk of developing cancers by inhibiting the pro-inflammatory signaling pathway. Apart from that, it is also known to cause cancer cells to self-destruct via apoptosis. Many Feverfew extracts are available, but those with high parthenolide content may be necessary to provide the anti-cancer effect.

\section{Red Clover}

The Red Clover Trifolium pretense was favored by Hippocrates for its medicinal properties. It has a long history of being used for treating coughs and colds and other respiratory ailments and to purify blood and get rid of kidney stones. It was one of the herbs found to be having cancer-fighting property when veterinarian John Hoxsey accidentally came across his horse getting cured of a tumor by grazing in an area where it grew.

Its use in cancer treatment is based on the isoflavones such as Genistein found in the plant. They act as phytoestrogens and down regulate the action of the estrogen generated in the body. Since this hormone is implicated in a majority of breast cancers as well as endometrial and ovarian cancers in women and prostate and testicular cancers in men, phytoestrogens play a significant role in controlling their growth and spread.

\section{Astragalus}

It is a Chinese medicinal herb commonly used in their medical tradition as an anti-aging agent. The shortening of the telomeres that protect our DNA strands is a sign of cellular aging. The bioactive compounds in this herb such as cycloastragenol are known to protect the telomeres. The same action may be beneficial in preventing cancers that may be initiated by damaged cells.

Astragalus stimulates the immune system and increase interferon production. Clinical studies have shown that using this herbal extract adjuvant to radiotherapy results in better outcome and significant increase in survival times.

\section{Chaparral}

This North American plant Larrea tridentate has a long history of being used in various medicinal preparations of the Native Indians. The plant is called creosote bush because of its characteristic smell. Chaparral tea has been used to treat various types of cancers including colon cancer, ovarian and cervical cancer and prostate cancer.

This bitter brew contains nor-dihihydroguairetic acid (NDGA) which has anti-tumor activity. It is thought to destroy cancer cells by inhibiting their utilization of glucose. This literally starves them to death.

\section{Echinacea}

The extract of purple coneflower Echinacea purpurea is a popular cold and flu remedy. The immune-boosting property of this plant has long been exploited by Native Americans, and extensively studied by modern researchers.

The polysaccharides in echinacea, especially arabinogalactan, have been found to activate macrophages and increase T-cell activity as well as the production of interferon. The plant extract contains other bioactive substances such as caffeic acid and flavonoids. Several studies have shown it to be effective against brain tumors.

\section{Sweet Wormwood}

This herbaceous medicinal plant Artemisia annua is different from the European herb Artemisia absinthium used in alcoholic drinks. Sweet wormwood is an Asian native that has been used in Chinese herbal medicine to treat many ailments including malaria. Now it has been found to be effective in treating breast cancer.

Artemisinin found in the plant extract selectively targets cancer cells and destroys them without affecting normal cells because of the higher iron intake by cancer cells. When artemisinin is combined with iron-carrying transferrin, it gets readily absorbed by the cancer cells. Once it gets inside the cells, artemisinin does its work.

The idea that simple plants, herbs, and foods can have anti-cancer effects is sometimes a controversial subject. Although many people have experienced their benefits, there are still a great many skeptics. For every person who believes that herbs and plants can slow or even kill cancer cells, there is another who will only believe in the merits of chemotherapy.

Although there is still work to do in the area of scientific research, a huge amount of research has already been completed. We are going to present that information here. Skeptics rarely change their minds unless it happens to them directly. However, for those of you who are interested in this information and wish to apply it to your life, we have compiled a list of the top 20 herbs and medicinal plants that have had scientific research applied to them and received positive outcomes in the area of fighting cancer. As always, keep in mind that this is not meant to be medical advice; it's simply information for you to consider.

The studies listed below show that all herbs work in a different manner. Some have what is called "immunomodulators," which means that they stimulate your own immune system to fight cancer cells. Others have cytotoxic action, meaning that they do kill cancer cells, but they can also kill healthy cells and should only be used under the supervision of a doctor or herbalist. Still others, such as Milk thistle, have been found by studies to kill only cancer cells. These, of course, are the most desirable herbs.

Please take note about the way certain terms are used here. Anticancer is a broad word that can be broken down into three parts:

-Anti-tumor - shown to be toxic to tumors in animal studies 
- Cytotoxic - shown to fight tumors in laboratory cell cultures (in vitro)

- Anti-cancer - shown to fight tumors in humans

Cancer usually takes years to develop, so prevention is preferable to any treatment. Avoid all known carcinogens such as tobacco, excessive alcohol, processed foods, and exposure to chemicals. A plant based diet can help protect you from cancer as plants are rich in antioxidant and anti-inflammatory compounds, both of which are powerful cancer fighters.

\section{Burdock Root}

Burdock root (arctium lappa) is native to northern Asia and Europe. Historical texts described burdock root as a blood purifier. Ancient Chinese physicians would treat venereal diseases, kidney problems, skin conditions, and respiratory issues with this root. It was well known in Medieval Europe for treating gout, pneumonia, and arthritis. Recent research shows that burdock root is very effective at removing the cancer causing toxins that accumulate in our digestive systems when certain foods are not properly digested. There are several anti-cancer herbal compounds that have used burdock root as a base including "Flor-Essence," and "Essiac." In fact, one anti-cancer formula sold and marketed in 1919, called "Hoxsey," was burdock root.

Some herbalists state that burdock root can stop cancer cells from metastasizing. It is often used in India and Russia for the treatment of cancer. Burdock can be used safely, except for those who are on potassium lowering diuretic therapy, as it contains fairly high quantities of potassium.

\section{Grape Seed}

Several studies have shown that the phytochemicals in grape seeds have anti-tumor or strong potential cancer preventative abilities that can be isolated from the seeds themselves. Proanthocyanidins are especially worth noting, as these have been found to stop pancreatic cancer cells from spreading or migrating.

One research team at the University of Colorado showed evidence that grape seed extract was effective against colorectal cancer. Extract from grape seed extract induced the death of these types of cancer cells. In fact, the more advanced the cancer cells were, the better the grape seed extracts seemed to work at limiting the growth and survival of cancer cells. Grape seed extract was not only causing the death of cancer cells, but it did not harm healthy cells.

The proanthocyanidins in grape seed extract have also been reported to inhibit the creation of new blood vessels and to arrest the growth of colon tumors. Proanthocyanidins accumulate in large amounts in the colon because they are not well absorbed in the stomach. This is good news, as the proanthocyanidins can stop cancer cells more efficiently as they build up in the colon.

\section{Goldenseal}

Goldenseal is native to North America and has been used for hundreds of years by the Native American people to treat numerous infections, including infections of the mouth, throat, or gums. This plant is related to buttercups and was once so popular that it was harvested almost to the point of extinction! Goldenseal is a key ingredient in many mouthwashes and toothpastes due to its antibacterial compounds which prevent tooth decay and kill bacteria. The powerful compound in goldenseal, berberine, has antibiotic compounds. This herb is currently undergoing scientific tests to see if it can lower cholesterol, and by lowering cholesterol levels, it lowers the risk of developing certain types of cancer. One of the main problems with manufactured goldenseal supplements is that there is little or no oversight as to how much of the active ingredient each capsule contains. Care should be taken with goldenseal and it is best used under the supervision of a doctor or herbalist as high doses can become toxic.

\section{Clove}

Clove is actually the dried flower buds from trees native to India, Pakistan, Zanzibar, and Madagascar. Clove oil from cloves (Eugenia aromaticum or Eugenia caryophyllata) has been studied for their ability to improve immune function, which means it offers cancer protection or prevention.

Clove oil has antioxidant compounds that have the potential to act as an anticancer agent, according to the American Pharmaceutical Association's Practical Guide to Natural Medicines. Clove oil has the highest concentration of antioxidant activity of any single ingredient tested by the ORAC.

The American Cancer Society says that there is currently insufficient evidence to show that clove oil can either treat or prevent cancer, however its high antioxidant levels show that it is a powerful supplier of anthocyanins, which are known to stop the growth of tumors, as well as kill existing cancer cells.

\section{Licorice Root}

One of the oldest botanical plants around, licorice root (glycyrrhiza glbra) has been used by Ancient Chinese medicine as an anti-virus, anti-inflammatory, and anti-ulcer agent. DNA damage is often done by carcinogens, and licorice root provides protection from that type of damage. Licorice root also contain polyphenols that encourage apoptosis (automatic death) in cancer cells. Licorice regulates the production of hormones from your adrenal glands, and it reduces stress chemicals. Chronic stress often triggers the growth of cancer cells.

Licorice root stops the proliferation of breast cancer cells in humans, according to a study done in South Korea. It modulates the expression of $\mathrm{Bcl}-2 / \mathrm{Bax}$ apoptotic regulatory factors.

Licorice root has been linked to some side effects such as high blood pressure, and muscle weakness. This is another herb that, if you plan to consume larger quantities on a regular basis, you should do so only under the supervision of your doctor or herbalist.

\section{Bloodroot}

Bloodroot is native to Canada, eastern North America, and Nova Scotia. It was used by indigenous peoples as an aid for respiratory problems. Externally, it has been used as a home remedy for 
skin cancer and is possibly the most well-known anti-cancer herb around. There have been several publications showing that bloodroot has the potential to be a powerful anticancer agent. Bloodroot (sanguinaria canadensis) has been shown in several studies to have consistent anti-neoplastic activity; it can shrink tumors, and has shown itself to be useful when dealing with sarcomas. The sap is toxic if consumed in anything more than minute amounts but all parts of the plant can be used externally. It's often used in naturopathic treatments for skin cancer.

The best evidence for bloodroot herbal remedies is that it seems to work best on skin cancer, however it's best to have a doctor's support in ensuring that all the cancer cells have been destroyed.

\section{Barberry}

This plant has been used for more than 3,000 years in Ayurveda. Mostly used for fevers, fatigue, and stomach upsets, barberry (berberis vulgaris) has recently been recognized as a powerful anti-cancer herb.

Barberry has strong anti-inflammatory, antibiotic, and antioxidant properties. It is often used interchangeably with goldenseal, as the two plants share a similar composition. Recent studies show that barberry improves immune functions and can lower high blood pressure.

According to Ayurvedic tradition, barberry is an effective treatment for liver tumors. One study done in Taiwan shows that when barberry is administered in high doses to cancer patients over time, the alkaloid in barberry, berberine, killed cancer cells in tests done with humans.

Barberry is another of the ingredients in the Hoxsey herbal formula marketed in 1919 as a cancer treatment.

Tea

Considered by many cultures to have healing and preventative compounds, including against cancer, tea (camellia sinensis) has been the focus of several scientific studies recently. The results of these studies vary somewhat, but tea was found to have disease preventing compounds.

One study performed by the USDA in 2006 found that tea had more than 700 different compounds, many of which were already known for their ability to fight disease. Among these compounds known to fight disease are polysaccharides, flavonoids, certain vitamins, and amino acids. All varieties of tea, including green white, black, and oolong, were found to contain high levels of antioxidants, which are powerful anti-aging and anti-tumor agents.

Consuming tea regularly can greatly improve the intestinal balance of healthy bacteria by increasing beneficial microorganisms while ridding the body of harmful bacteria. Tea has high levels of vitamin $\mathrm{C}$, which is known to fight cancer causing free radicals. Tea has a low glycemic index and has strong cleansing properties. Regular consumption of tea has long been associated with the prevention of diabetes and heart disease.

\section{Onion}

Onion (allium cepa) has a high antioxidant activity and is associated with a variety of pharmacological items including being antiinflammatory, antibiotic, and anti-carcinogenic. One study showed greater antioxidant activity of older rats that were fed onion.

There is a definite relationship between the consumption of onions and the risk of common cancers. Researchers from the Italian Mario Negri Institute for Pharmacological Research compiled data from both Italian and Swiss controlled studies and multivariate logistic regression models for onion consumption and cancer rates. The risk rates vary, but onions lowered the risk of colorectal cancer, ovarian cancer, renal cell cancer, prostate cancer, esophageal cancer, mouth cancer, and breast cancer.

Onions are high in polyphenols, which prevent diseases, including cancer. Onions are also high in antioxidants, which are also known cancer fighters. These popular veggies also contain a compound called quercetin, which has been shown to decrease cancer tumor cells.

\section{Dandelion}

There has been strong evidence lately that dandelion, that weed in your garden, can inhibit the development and growth of numerous types of cancer, including stopping their metastasizing capabilities. Read more how to harvest dandelion.

Dandelion leaves have long been used by Macedonian phytomedicine and Ayurvedic practitioners to treat abscesses, water retention, tumors, and cysts. A study done in 2008 provided some scientific proof that dandelion (taraxacum officinale) extracts had anti-cancer compounds. During this study, three extracts were made from mature dandelion leaves, roots, and flowers. All parts were tested for their activity on the progression of tumors. This study showed that the leaf of this common weed blocked the growth of breast cancer cells and the invasions of prostate cancer cells. Dandelion root extract stopped the invasion of breast cancer cells.

The flower of this plant has high antioxidant compounds as a Canadian study showed in 2005. This study showed that dandelion extract stopped hydroxyl and superoxide radicals, which means that dandelion has the potential to be a powerful, yet novel, cancer fighter.

\section{Foxglove}

There have been several studies done regarding foxglove and its possible anti-cancer capabilities. There are two varieties of this plant, Digitalis purpurea L and Digitalis lanata ehrh. These two have been looked at for their vitro cytotoxicity after polypeptide isolation and extraction. Both have been shown to have strong anti-tumor possibilities.

A study performed in Spain in 2003 look at the cytotoxic activity from the leaves of the strain D. purpurea against human cancer cells. The extracts used (C50 $0.78-15 \mathrm{ug} / \mathrm{ml}$ ) especially the methanolic extract, had high cytotoxic action. 
Acteoside, which can be extracted from the leaves of this common flower, have a hepatoprotective effect from a toxin called aflatoxin B1. This makes this plant cytotoxic and a strong potential chemopreventative possibility.

It's the chemical in the foxglove flower called digoxin that is known to slow the spread of breast cancer cells, as well as prostate cancer by as much as 24 percent. Keep in mind this is an extract that has been removed professionally. NEVER consume foxglove from your garden or from any unknown or untrustworthy source.

It should be noted that foxglove is quite poisonous, so it must be used only with the advice and supervision of a doctor or herbalist.

\section{Graviola}

Graviola, also known by the name of its fruit, sour sop, has received a great deal of attention lately because of its promising compounds as possibly being a natural anti-cancer agent. Graviola (Annona muricata) is an evergreen tree that is used as a remedy for coughs, bacterial and parasite infections, herpes, and arthritis.

A great deal of the excitement over this fruit came from a study done in 2012 in Nebraska at the Department of Biochemistry and Molecular Biology that showed that sour sop was effective against pancreatic cancer cells. These types of cells are extremely resistant to conventional therapies. By inhibiting several signaling pathways that pancreatic cancer cells use to regulate their survival and metastatic lifecycle, graviola kills these cells when they are unable to complete their normal function. Once they are inhibited in this manner, the rate at which pancreatic cancer cells spread to other organs to make new cancers becomes incredibly slow.

Still another study done in Virginia at Virginia Tech, found that sour sop extracts also stopped the growth of breast cancer cells. Sour sop extract also inhibited the growth of EGFR. You should note that cell mutation result in the overproduction of EGFR has been strongly connected to several cancers.

You might have heard a great deal of controversy over sour sop and it's possibly hyped up claims of its cancer killing abilities.

\section{Milk Thistle}

There have been several studies that have shown that the extract of the seed coating of milk thistle has anti-cancer effects. Milk thistle (silybum eburneum or silybum marianum) has an active ingredient in their seeds contain a wide mixture of flavonolignans such as silibinin, silidianin, silicristin, and isosilibinin. Of all of these, the main active ingredient, silibinin has shown the strongest in vitro effects against human prostate cancer cells, human colon cancer cells, human lung cancer cells, and estrogen dependent and estrogen independent human breast cancer cells. Silymarin appears to have no adverse effects even when given in high oral doses of $1 \mathrm{~g}$ per kilogram of weight in dogs, and $20 \mathrm{~g} / \mathrm{kg}$ in mice.

Two studies done recently at the University of Colorado Cancer Center Studies have shown that silibinin, the active ingredient in the seeds from milk thistle plants, has efficacy from both UVB and UVA induced skin cancer and photo-aging. These studies, done in 2012 and 2013, showed the amazing ability of silibinin to selectively kill skin cells mutated by UVA but were nontoxic to healthy skin cells. It also accelerated the repair of UVB damaged noncancerous cells.

\section{Mistletoe}

The extracts from mistletoe (viscum album) and the products that come from this extract, especially iscador, is one of the most commonly used oncological drug in Europe. It's been used as an overall treatment for cancer for years. One randomized and nonrandomized matched pair study that involved more than 10,000 cancer patients, iscador was shown to prolong the survival time of cancer patients.

Amazingly, this study showed that the overall survival time of the group that took iscador was as much as 40 percent longer when compared to the control group.

Mistletoe extract is also known as having a better survival among cancer patients and there are numerous studies that show the positive effects of this plant on cancer patients.

\section{Saffron}

Saffron contains a carotenoid compound called crocetin. The results of studies done, both in vivo and in vitro, show that this compound has the potential to be a strong anti-tumor agent. Saffron was found in another study to inhibit skin cancer in mice.

New research shows that this popular spice has a powerful chemo-preventative effect against liver cancer in tests done with animals. This study came out in September of 2011 in the journal Hepatology, and was done by the American Association for the Study of Liver Diseases.

\section{References}

1. Dragan Jovanov, Macedonian herbal plant, nutrition, sport and health, Lumbert publiching, 2016

2. Dragan Jovanov, Macedonian modern phytomedicine, Matica Makedonska, 2016, Macedonia

\section{Dragan Jovanov, Herbal plant, Macedonia}

4. Dragan Jovanov, Aromatic herbal plant, MIT University, Skopje- Macedonia, 2015

5. Cura Holistic Health clinic, Shannon Burford, Australia 